\title{
Recomendaciones para la rehabilitación pulmonar en pacientes con COVID-19
}

\author{
Recommendations for pulmonary rehabilitation in patients with COVID-19 \\ Jhonatan Betancourt-Peña ${ }^{1 *}$ orcid.org/0000-0002-7292-7628 \\ Diana Suaza-Casañas² orcid.org/0000-0003-2839-1245 \\ Johana Carolina Pazmiño-Ordoñez ${ }^{3}$ orcid.org/0000-0003-0004-3396 \\ Carolina Hurtado-Pantoja ${ }^{4}$ orcid.org/0000-0002-0665-0456 \\ Johan Andres Parra-Liévano5 orcid.org/0000-0003-1551-279X \\ Lina Jimenez ${ }^{6}$ orcid.org/0000-0002-6423-6771 \\ Mónica Lizeth Márquez-Flórez orcid.org/0000-0002-0984-3130 \\ Rosa Virginia Mora-Guerra ${ }^{8}$ orcid.org/0000-0002-1817-6114
}

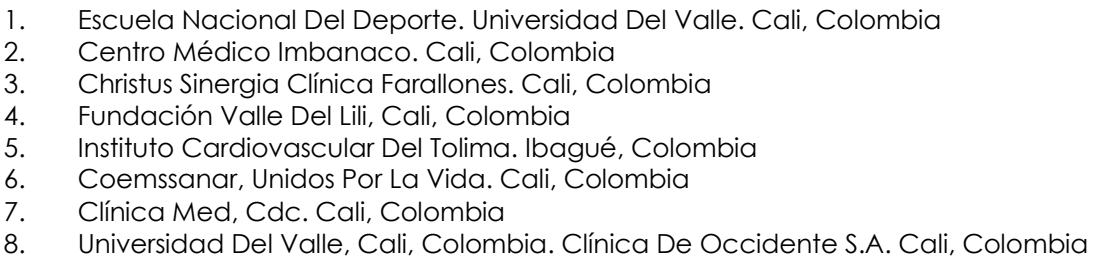

Fecha de recepción: Agosto 25 - 2020

Fecha de revisión: Febrero 23 - 2021

Fecha de aceptación: Diciembre 20 - 2021

Betancourt-Peña J, Suaza-Casañas D, Pazmiño-Ordoñez JC, Hurtado-Pantoja C, Parra-Lievano JA, Jimenez L, Márquez-Flórez ML, Mora Guerra RV. Recomendaciones para la rehabilitación pulmonar en pacientes con COVID-19. Univ. Salud. 2022;24(1):76-84. DOI: https://doi.org/10.22267/rus.222401.260

\section{Resumen}

Introducción: La COVID-19 es una enfermedad que desencadena infecciones respiratorias graves como el síndrome respiratorio agudo severo, que puede requerir atención prolongada intra y extrahospitalaria. Objetivo: Brindar recomendaciones y pautas para una correcta evaluación y tratamiento del paciente con COVID-19 mediante la rehabilitación pulmonar. Materiales y métodos: Revisión de la literatura en las bases de datos MEDLINE, PEDRO, Scielo y en Google Scholar, a partir de las palabras clave: COVID-19, Síndrome de Dificultad Respiratoria del Adulto, Enfermedades pulmonares, Rehabilitación, pruebas de función respiratoria, Unidad de cuidado intensivo; seleccionando, aquellos artículos que presentaran información sobre rehabilitación en pacientes con COVID-19 y otras alteraciones con compromiso similar como el Síndrome Respiratorio Agudo y las Enfermedades Pulmonares Intersticiales Difusas. Resultados: Para realizar una intervención en paciente con COVID-19 u otra alteración respiratoria como Síndrome de Dificultad Respiratoria del Adulto y Enfermedad Pulmonar Intersticial Difusa, es necesario realizar un adecuado diagnóstico de la enfermedad, una minuciosa evaluación integral y una intervención basada en las necesidades de cada individuo. Conclusiones: El paciente intrahospitalario puede recibir intervención enfocada a evitar el deterioro funcional y una vez son dados de alta la rehabilitación pulmonar extrahospitalaria podría ser una alternativa eficaz en el tratamiento para los pacientes.

Palabras clave: COVID-19; síndrome de dificultad respiratoria del adulto; enfermedades pulmonares; rehabilitación; pruebas de función respiratoria; unidad de cuidados intensivos. (Fuente: DeCS, Bireme).

\begin{abstract}
Introduction: COVID-19 is a disease that triggers serious respiratory infections such as severe acute respiratory syndrome, which requires treatment on an inpatient and outpatient basis. Objective: To provide recommendations and guidelines for the appropriate assessment and treatment of COVID-19 patients through pulmonary rehabilitation. Materials and methods: A literature review using MEDLINE, PEDRO, Scielo and Google Scholar databases and the keywords: COVID-19, Adult Respiratory Distress Syndrome, Pulmonary diseases, Rehabilitation, Respiratory function tests, Intensive Care Unit. Articles that described information regarding rehabilitation in COVID-19 patients and other similar dysfunctions such as Acute Respiratory Syndrome and Diffuse Interstitial Lung Diseases. Results: It is necessary to carry out an appropriate disease diagnosis, a detailed integral assessment, and an intervention based on the needs of each patient in order to intervene with either COVID-19 patients or cases with other respiratory dysfunctions such as Adult Respiratory Distress Syndrome and Diffuse Interstitial Lung Disease. Conclusions: Inpatients can be subjected to an intervention focused on avoiding functional failure. Once they are discharged, outpatient pulmonary rehabilitation could be an effective treatment alternative for these patients.
\end{abstract}

Keywords: COVID-19; respiratory distress syndrome; lung diseases; rehabilitation; respiratory function tests; intensive care units. (Source: DeCS, Bireme).

*Autor de correspondencia

Jhonatan Betancourt Peña

e-mail: johnnatanbp@hotmail.com 


\section{Introducción}

El SARS-CoV-2 hace parte de la familia de los coronavirus, los cuales son virus que emergen de manera periódica en diferentes lugares del mundo y que desencadenan infecciones respiratorias que pueden ir desde el resfriado común hasta enfermedades más graves como el síndrome respiratorio de Oriente Medio (MERS) y el síndrome respiratorio agudo severo (SRAS)(1). El SARS-Cov-2 presenta un cuadro clínico compatible con una infección respiratoria, que dependiendo de los factores de riesgos y severidad puede requerir atención durante tiempos prolongados y posteriormente desencadenar secuelas importantes en factores como debilidad adquirida de paciente crítico, intolerancia al ejercicio, disfunción respiratoria, disfagia, disfonía y disminución del nivel de calidad de vida ${ }^{(2)}$.

Teniendo en cuenta la gran limitación funcional y psicosocial que presenta el paciente, la rehabilitación pulmonar se debe integrar dentro del tratamiento individualizado con el objetivo de reducir síntomas, optimizar el estado funcional, aumentar la participación y reducir los costes sanitarios mediante la estabilización o la reversión de las manifestaciones sistémicas de la enfermedad(3). Con base en lo anterior este artículo tiene como objetivo brindar recomendaciones y pautas para una correcta evaluación y tratamiento del paciente con COVID-19 mediante la rehabilitación pulmonar.

\section{Materiales y métodos}

Se realizó una revisión de la literatura mediante búsqueda bibliográfica en las bases de datos MEDLINE, PEDRO, Scielo y en el buscador de Google Scholar. La búsqueda se realizó a partir de palabras clave: Infecciones por coronavirus, Síndrome de Dificultad Respiratoria del Adulto, Enfermedades pulmonares, Rehabilitación, Pruebas de función respiratoria, Unidad de cuidado intensivo, en español, y Coronavirus infections, Respiratory Distress Syndrome, Adult, lung diseases, Rehabilitation, Respiratory Function Tests, Critical Care en inglés; seleccionando aquellos artículos que presentaran información relevante sobre rehabilitación en pacientes con COVID-19 y otras alteraciones respiratorias con compromiso similar como el Síndrome de Dificultad Respiratoria del Adulto (SDRA) y la enfermedad pulmonar intersticial difusa (EPID). El periodo de la búsqueda se limitó a estudios publicados entre el año 2000 al 2020, y se filtró la búsqueda para artículos en español e inglés utilizando las palabras clave de los descriptores en Ciencias de la Salud (DECS) y Medical Subject Headings (MeSH). Dicha búsqueda fue realizada entre los meses Abril - junio del 2020, se recuperó 57 artículos de los cuales se excluyeron 25; los 32 artículos restantes presentaban información relevante para la revisión del tema.

\section{Resultados}

Previo a la revisión de las recomendaciones para llevar a cabo intervenciones en rehabilitación pulmonar de las personas con COVID-19, se considera importante mencionar las alteraciones que pueden presentar en los sistemas relacionados con el componente respiratorio (Tabla 1).

Pautas para los programas de Rehabilitación Pulmonar en contexto de Pandemia por Covid-19 Para la intervención de los pacientes, se tienen dos momentos; el intrahospitalario durante el proceso agudo de recuperación, y el extrahospitalario o tras el alta, en donde se van a evidenciar las secuelas a mediano y largo plazo mencionadas anteriormente. A continuación, se presentan las estrategias de intervención en rehabilitación pulmonar que deben llevarse a cabo con los pacientes con COVID-19.

\section{Pautas de rehabilitación pulmonar intrahospitalaria para pacientes con COVID-19}

El objetivo a corto plazo de la rehabilitación pulmonar es aliviar la disnea al igual que la ansiedad y la depresión, mientras que el objetivo a largo plazo es preservar la funcionalidad del paciente al máximo, mejorar su calidad de vida y facilitar su regreso a la sociedad(15). En comparación con la rehabilitación general para pacientes con enfermedades crónicas, la característica más distintiva de la rehabilitación para pacientes con COVID-19 es el alto grado de transmisión de la enfermedad. Por lo tanto, las actividades que pueden aumentar el riesgo de infección, como la tos instruida, el entrenamiento de espiración y la compresión traqueal, deben minimizarse ${ }^{(15)}$.

Los pacientes con COVID-19 que padecen enfermedades pulmonares crónicas a menudo tienen secreciones excesivas de las vías respiratorias, por lo cual se deben realizar ejercicios de espiración además de los ejercicios generales de limpieza de las vías respiratorias, para facilitar la excreción de esputo y reducir el agotamiento debido a la tos(15). 
Tabla 1. Sistemas Afectados en la COVID-19

\begin{tabular}{|c|c|c|}
\hline Sistema & Alteraciones encontradas periodo agudo & Alteraciones encontradas periodo tardío \\
\hline Cardiovascular & $\begin{array}{l}\text { Arritmia, miocarditis, síndrome coronario agudo, } \\
\text { tromboembolismo venoso, shock cardiogénico, falla } \\
\text { cardiaca( }{ }^{4)} \text {. }\end{array}$ & $\begin{array}{l}\text { Disminución de la capacidad aeróbica en el período } \\
\text { temprano de rehabilitación, disminución en el } \mathrm{VO}_{2} \text { máx. y } \\
\text { de la distancia recorrida en la prueba de marcha de } 6 \\
\text { minutos, anemia y desacondicionamiento físico(5). }\end{array}$ \\
\hline Respiratorio & $\begin{array}{l}\text { Debilidad muscular respiratoria que desencadena } \\
\text { defecto restrictivo de la función pulmonar(6), apnea del } \\
\text { sueño(7) y cambios fibróticos pulmonares }\end{array}$ & $\begin{array}{l}\text { Al año del alta hospitalaria; en algunos pacientes } \\
\text { persisten síntomas como tos, aumento de producción de } \\
\text { esputo, dificultad para respirar, sibilancias ocasionales, } \\
\text { fibrosis pulmonar( }(9) \text {, entre otros. } \\
\text { Debilidad de los músculos respiratorios con valores } \\
\text { Pimax y Pemax por debajo de } 80 \mathrm{cmH}_{2} \mathrm{O} \text { a los } 6 \text { meses }(10) \text {. } \\
\text { La debilidad de los músculos espiratorios podría } \\
\text { conducir al atrapamiento de aire (como se refleja por un } \\
\text { aumento de RV por encima de } 120 \% \text { del predicho en } \\
\text { algunos pacientes), mientras que la debilidad de los } \\
\text { músculos inspiratorios puede conducir a atelectasia(1). }\end{array}$ \\
\hline $\begin{array}{l}\text { Músculo } \\
\text { esquelético }\end{array}$ & $\begin{array}{l}\text { Dolor musculoesquelético variable, debilidad } \\
\text { profunda, fatiga, función física limitada, debilidad } \\
\text { neuromuscular, disfagia, requerimiento de intubación } \\
\text { prolongada de algunos pacientes(11), miopatía por } \\
\text { corticoides }(9) \text {, necrosis vascular de la cabeza } \\
\text { femoral }(12) \text {. }\end{array}$ & $\begin{array}{l}\text { Limitación para realizar actividades de la vida diaria y } \\
\text { dolor muscular crónico. }\end{array}$ \\
\hline Neurológico & $\begin{array}{l}\text { Emocionales: Estrés psicológico, depresión, y grandes } \\
\text { problemas de sueño(7). } \\
\text { Encefalitis: Autoinmunidad en individuos susceptibles } \\
\text { (neuroinmunopatología inducida por virus) y/o } \\
\text { replicación viral, lo que causa daño directamente a las } \\
\text { células del SNC (neuropatología inducida por virus), }{ }^{13} \\
\text { Parálisis flácida transitoria(13), Esclerosis Múltiple } \\
(\mathrm{EM})^{(15) .}\end{array}$ & $\begin{array}{l}\text { Estrés postraumático crónico caracterizado por ansiedad } \\
\text { elevada, estrategias de afrontamiento deterioradas y } \\
\text { sesgo atencional al dolor y déficit funcional; todos estos } \\
\text { factores pueden exacerbar el dolor y causar deterioro } \\
\text { funcional(14). }\end{array}$ \\
\hline
\end{tabular}

La hipoxemia durante el ejercicio se considera la indicación para el requerimiento de oxigenoterapia (saturación de oxígeno capilar periférico $\left[\mathrm{SpO}_{2}\right] \mathrm{de}$ $88 \%-90 \%$ o una reducción relativa de $2 \%-5 \%$, con una duración de 0,5-5,0 min). El objetivo de la oxigenoterapia es ajustar el caudal de oxígeno para mantener la $\mathrm{SpO}_{2}$ dentro del rango del $90 \%$ al $92 \%$. Para aumentar el efecto del ejercicio, la tasa de flujo de oxígeno se puede incrementar de acuerdo con la intensidad del ejercicio para mantener la $\mathrm{SpO}_{2}$ en aproximadamente el 95\%(15). Toda rehabilitación debe llevarse a cabo bajo la premisa de seguridad. En caso de que un paciente muestre una $\mathrm{SpO}_{2}<88 \%$ o desarrolla síntomas, como palpitaciones, sudoración, opresión en el pecho y dificultad para respirar, que el clínico considera inadecuados para la rehabilitación, entonces el programa de rehabilitación debe finalizar inmediatamente.

Para casos leves y moderados, las intervenciones de rehabilitación deben introducirse lo antes posible. Por el contrario, para casos graves y críticos, las medidas que salvan vidas deben priorizarse cuando la condición del paciente es inestable o la enfermedad aún está progresando(15). El contenido del programa de Rehabilitación Pulmonar debe estar conformado por:

a. Ejercicios aeróbicos: caminar, caminar rápido, entre otros; comenzando con baja intensidad y mejorando gradualmente la intensidad y la duración, de 3 a 5 veces por semana, de 20 a 30 minutos cada vez.

b. Entrenamiento de fuerza: se recomienda el entrenamiento de resistencia progresivo. La carga de entrenamiento de cada grupo muscular objetivo es 8-12 repeticiones, 1-3 grupos musculares iniciando con miembros inferiores para permitir la deambulación posterior en un tiempo estimado de 20 minutos. El intervalo de entrenamiento de cada grupo es de 2 minutos, de 2 a 3 veces por semana, y la carga de entrenamiento aumenta entre un $5 \%$ y un $10 \%$ cada semana.

c. Entrenamiento de equilibrio: Los pacientes con disfunción del equilibrio deben participar en el entrenamiento del equilibrio, incluido el entrenamiento del equilibrio en posición bípeda con apoyo y posteriormente sin apoyo. 
d. Entrenamiento respiratorio: si el paciente tiene síntomas, como dificultad para respirar, sibilancias y dificultad para expectorar después del alta, entrenamiento en modo respiratorio, como manejo de la posición corporal, ajuste del ritmo respiratorio, tracción del ejercicio de respiración del grupo muscular respiratorio y el entrenamiento de expectoración debe organizarse en combinación con los resultados de la evaluación.

Se recomienda dentro del programa de rehabilitación pulmonar, tener en cuenta las siguientes condiciones(16):

\section{Criterios de exclusión para dar inicio al programa:}

1. Frecuencia cardíaca $>100$ latidos / min.

2. Presión arterial $<90 / 60 \mathrm{mmHg} 0>140 / 90 \mathrm{mmHg}$.

3. $\mathrm{SpO}_{2}$ menor a $90 \%$ con soporte de oxígeno suplementario.

4. Otras enfermedades no aptas para el ejercicio.

\section{Criterios de interrupción del ejercicio:}

1. Fluctuaciones en la temperatura corporal $>37,2$ ${ }^{\circ} \mathrm{C}$.

2. Aumento de los síntomas respiratorios, fatiga y falta de alivio después del descanso.

3. Detener inmediatamente la actividad y consultar a un médico si aparecen los siguientes síntomas: opresión en el pecho, dolor en el pecho, dificultad para respirar tos severa, mareos, dolor de cabeza, visión borrosa, palpitaciones del corazón, sudoración, inestabilidad y otros síntomas, así como arritmias no controladas durante la monitorización.

\section{Pautas para pacientes que acuden a un programa} de Rehabilitación Pulmonar de consulta externa Previo al inicio de cada sesión, se recomienda aplicar un pequeño cuestionario sobre síntomas respiratorios, el cual se puede entregar al paciente para que lo tenga durante todo el programa de rehabilitación pulmonar (RP) ${ }^{(17)}$.

Lista de chequeo de síntomas a preguntar a los pacientes previo inicio de sesión de rehabilitación:

- ¿Ha presentado fiebre en las últimas 24-48 horas?

- ¿Existe un empeoramiento de su ahogo en las últimas 24-48 horas?

- ¿Existe cambio en su expectoración en cantidad, color esputo en las últimas 24-48 horas?
- ¿Presenta alguna sintomatología de nueva aparición en las últimas 24-48 horas: síntomas digestivos, cansancio, malestar general?

- Recordar la higiene de manos antes de entrar en la sala y al salir.

Debido a que en la Rehabilitación Pulmonar (RP) se pueden generar aerosoles, se recomienda que el paciente use de mascarilla quirúrgica, desde la llegada a la unidad. Si el paciente se muestra particularmente incómodo con el uso de mascarilla quirúrgica durante la práctica del ejercicio, el uso de pantallas faciales puede ser una alternativa a valorar.

Los pacientes con necesidad de oxigenoterapia durante el entrenamiento deberán ponerse mascarilla quirúrgica sobre la interfase utilizada. Esto aplica igualmente para la oxigenoterapia de alto flujo nasal.

\section{Evaluación clínica pre rehabilitación en COVID 19}

Dicha evaluación corresponde al primer contacto con el paciente y es realizada por el especialista en Rehabilitación Cardiaca y Pulmonar o Fisioterapia Cardiopulmonar encargado del programa de rehabilitación. Se debe realizar:

- Anamnesis cardiorrespiratoria.

- Examen físico: examen integral con énfasis en el sistema cardiorrespiratorio y osteomuscular, patrón respiratorio, utilización de los músculos accesorios y valoración completa del tórax y de los músculos respiratorios, cualidades físicas coordinativas (equilibrio, coordinación, ritmo, etc.), las cualidades condicionales (fuerza, resistencia, velocidad y flexibilidad) y el trofismo muscular. Igualmente, se debe estratificar el riesgo de entrenamiento físico y el riesgo de caída(17).

\section{Evaluación de la capacidad funcional}

Para esta evaluación se recomienda realizar pruebas de ejercicio que se utilizan para identificar anormalidades funcionales no presentes en reposo, de tal manera que ayudan a cuantificar la verdadera severidad de la limitación funcional, así como para monitorizar los niveles de saturación de oxígeno, ayudar al diagnóstico diferencial de la limitación al ejercicio (cardiovascular, respiratoria, musculatura periférica). Adicionalmente, la prueba de capacidad funcional se utiliza para prescribir el componente de ejercicio del programa, y se considera el punto de base para evaluar los avances durante el seguimiento y al finalizar la rehabilitación. 
Para los pacientes dados de alta por COVID-19 se recomienda realizar la prueba de marcha de 6 minutos(18-21). La base fisiológica de esta prueba es que la distancia conseguida en un recorrido plano durante el tiempo estipulado (6 minutos) es una expresión de la capacidad del individuo para el ejercicio submáximo(22,23).

Las recomendaciones generales de la prueba son las siguientes:

\section{Instrucciones para el paciente}

- Usar tapabocas durante la ejecución de la prueba.

- Llevar ropa cómoda y calzado adecuado.

- Evitar comer durante 2 horas previa a la realización de la prueba.

- No se debe suspender medicamentos ni uso de broncodilatadores.

- El paciente debe tener claridad sobre la importancia del reposo 15 minutos previos al desarrollo de la prueba.

- Explicar al paciente la escala de Borg, el recorrido a seguir y el rol del personal de la salud.

- Durante las instrucciones de la prueba es necesario mencionarle al paciente que probablemente sienta falta de aire o cansancio, que está permitido disminuir la velocidad, detenerse o descansar si lo necesita.

- Si se detiene debe reiniciar la marcha tan rápido como sea posible, que no debe hablar en ningún momento de la prueba, a menos que tenga algún problema; y de ser así, será auxiliado inmediatamente.

\section{Instrucciones para el fisioterapeuta}

- El paciente debe descansar en una silla próxima al sitio de inicio de la prueba, en donde el fisioterapeuta debe revisar los signos vitales del paciente y de las posibles contraindicaciones para la continuación de la prueba.

- La determinación de la disnea y fatiga del paciente se hará de acuerdo con la escala de Borg.

- Desde el inicio hasta la finalización de la prueba, el examinador debe contar con todo el equipo necesario (cronómetro, escala Borg y medios para el conteo de las vueltas).

- Dar las siguientes instrucciones al paciente: “El objetivo de la marcha de seis minutos es caminar lo más rápido posible por 6 minutos sin correr, la distancia que usted va a recorrer va a ser desde esta línea de partida hasta el cono ubicado a 30 metros, dando la vuelta por detrás de este, en ningún momento usted debe correr; durante toda la prueba yo estaré acompañándolo; en caso de sentirse exhausto usted podrá parar o disminuir el ritmo durante el tiempo que considere necesario".

- Durante toda la prueba el examinador debe estar cerca del paciente, teniendo en cuenta las recomendaciones de distanciamiento social, se sugiere estar a 1,5 metros del paciente. En ningún momento el examinador debe ser la pauta para la marcha, debe estar lo suficientemente cerca para acompañar al paciente guardando la distancia necesaria para no interrumpir o afectar su marcha.

- Durante toda la prueba el examinador no debe motivar al paciente, solo podrá mencionar comandos recomendados cada minuto que transcurra la prueba.

- En el momento en que se termine la prueba, el examinador debe evaluar al paciente en el punto de finalización y de forma inmediata, la disnea y la fatiga con la escala de Borg, la saturación de oxígeno y la frecuencia cardiaca; posteriormente, debe iniciar la recolección de datos de acuerdo al protocolo estandarizado.

- Si durante la prueba el paciente se detiene, el examinador puede decirle, "usted puede descansar contra la pared si lo prefiere, luego continué caminando cuando usted se siente preparado", el examinador no debe parar de cronometrar el tiempo.

- Registre los datos de la historia clínica del paciente que solicita el formato de evaluación.

- Los intentos se deben repetir el mismo día en el mismo lugar y en las mismas condiciones, todo con el fin de evitar variables adicionales.

- Prueba de Práctica: Se recomienda la realización de dos pruebas de marcha, ya que la primera sirve como adaptación del paciente. La realización de una segunda caminata debe estar separada de la primera por lo menos 15 minutos si no es posible realizarla el mismo día, podrá hacerse al día siguiente, pero respetando el mismo horario de realización de la primera para evitar las variaciones circadianas de la función pulmonar. Administrar oxigeno suplementario a aquellos pacientes que presentaran una desaturación en el $6 \mathrm{MWT}$ u otra prueba de capacidad aeróbica $\geq \mathrm{a}$ $4 \%$ o que durante el ejercicio la $\mathrm{SpO}_{2}$ fuera $<90 \%$.

\section{Evaluación de la condición psicosocial}

Esta intervención es necesaria dado el impacto psicosocial de la enfermedad respiratoria crónica en 
el paciente y su familia, dentro de las escalas que se sugieren aplicar se encuentran:

- Escala de auto calificación de la depresión (SDS).

- Escala de ansiedad autocalificada (SAS)(19), Evaluación socio-familiar, Resumen e interpretación de la evaluación psicosocial(17). En su defecto la escala de ansiedad y depresión hospitalaria (HADS).

Esta valoración puede ser realizada por el profesional que hace la evaluación integral y se recomienda remisión a psicología en caso de necesidad.

\section{Durante la aplicación de las técnicas de Fisioterapia Respiratoria, se produce aerosolización, por lo cual se brindan las siguientes recomendaciones}

Instruir a los pacientes en técnicas con dispositivos de automanejo (ej.: Acapella, inspirómetro incentivador de flujo) que el paciente puede utilizar en su domicilio, para evitar generación de aerosoles en el gimnasio(20).

Otras técnicas de aplicación directa sobre el paciente deberán valorarse y hacer uso de la protección necesaria.

- Entrenamiento músculos inspiratorios: Preferiblemente instruir al paciente para que este tipo de entrenamiento lo realice en su domicilio. Si es necesario durante la sesión hospitalaria, utilizar filtros antivirales, que se pueden adaptar a algunos dispositivos, como el umbral (Threshold) ${ }^{(15,20)}$

- Entrenamiento fuerza: Se pueden aplicar ejercicios de fortalecimiento muscular con mancuernas, cintas elásticas, electro estimulación neuromuscular y aparatos gimnásticos, como es habitual en los programas de Rehabilitación Respiratoria(20).

- Cumplir distancia de seguridad, protección con mascarillas y guantes y lavado de manos antes y a la salida de cada aparato.

- Entrenamiento aeróbico: Mantener distancia de seguridad. Auto lectura de constantes $\left(\mathrm{FC} \mathrm{y} \mathrm{SpO}_{2}\right)$, mascarilla quirúrgica, el uso de paneles de metacrilato puede ser útil. Se deberá valorar el uso de guantes en caso de pulsioxímetro tipo dedil, con la alternativa de enfatizar el lavado de manos(20).

\section{Recomendaciones para la valoración de capacidad de esfuerzo y fuerza muscular}

Estas exploraciones son imprescindibles para los programas de rehabilitación respiratoria, entre otras cosas, para establecer la intensidad del entrenamiento físico.

Si las pruebas de esfuerzo no están disponibles, se recomienda utilizar la escala de Borg o la FC para indicar la intensidad del entrenamiento. Si las condiciones de seguridad lo permiten, las pruebas referidas pueden realizarse, con estas recomendaciones:

1. Prueba de marcha 6 minutos: Al momento de la revisión no se han realizado recomendaciones de expertos para este test. Creemos que mantener las medidas higiénicas, segregación y distancia seguridad, de protección individual para operador (EPI), y paciente (mascarilla quirúrgica, valorar guantes según tipo de pulsioxímetro), sería suficiente. Como alternativa a la mascarilla quirúrgica, se puede valorar uso de pantalla facial.

2. Prueba de esfuerzo cardiopulmonar: según normas de cada laboratorio. Es recomendable utilizar paneles de metacrilato para separar el cicloergómetro/tapiz rodante, del operador. Como alternativa a la prueba de esfuerzo cardiopulmonar, se puede utilizar el test de marcha Shuttle Walking Test, con iguales recomendaciones que la prueba de marcha de 6 minutos.

3. Test de valoración de fuerza muscular, periférica y respiratoria: según procedimiento habitual, con medidas de seguridad e higiene recomendadas anteriormente.

\section{Discusión}

Para esta revisión se evidenció que los pacientes que presentan SARSCoV2 pueden presentar alteraciones en diferentes sistemas que afecta considerablemente la condición física/emocional, capacidad funcional y calidad de vida relacionada con la salud de los pacientes, por ello, intervenciones como la rehabilitación pulmonar podría reducir significativamente el tiempo de hospitalización y mejorar el estado funcional de los pacientes.

Es claro que la rehabilitación pulmonar tiene como propósito reducir la disnea, controlar la ansiedad/depresión, evitar complicaciones, mejorar el desempeño de los sistemas muscular, cardiovascular y pulmonar junto con la calidad de vida relacionada con la salud. En cuanto a la evaluación de los pacientes es necesario caracterizar la población para identificar aspectos importantes, 
sobre todo teniendo en cuenta que es una enfermedad nueva y de la que se tiene escasa información sobre su impacto a largo plazo. El uso de escalas como Borg modificada y la mMRC permiten medir subjetivamente la sintomatología y son un punto de partida para comparar intervenciones; al igual que la valoración de la fuerza de grandes grupos musculares para la fatiga muscular(21,22).

También la medición de la capacidad funcional con la prueba de marcha de 6 minutos podría aportar información valiosa ya que se ha evidenciado disminución de la distancia recorrida en alteraciones respiratorias similares (23), a su vez, las mediciones de la ansiedad/depresión resultan de gran importancia para realizar intervenciones más integrales dirigidas no sólo al paciente, sino también a familiares e incluso cuidadores, teniendo en cuenta los diferentes contextos en los cuales se involucra, esto permite evaluar el nivel de calidad de vida de dichos pacientes ${ }^{(24) .}$

Por otra parte, el uso de nuevas escalas como la PostCOVID-19 Functional Status (PCFS) puede ser una alternativa para evaluar aspectos relevantes de la vida diaria durante el seguimiento posterior a la infección; a su vez, permite determinar objetivamente el grado de discapacidad(25).

La rehabilitación pulmonar ha evidenciado tener beneficios en diferentes enfermedades respiratorias crónicas como la Enfermedad Pulmonar Obstructiva Crónica (EPOC), Enfermedad Pulmonar Intersticial Difusa (EPID), e incluso condición post SDRA para disminuir la disnea, mejorar la capacidad funcional y la calidad de vida; no obstante pacientes con complicaciones ocasionadas posterior al SARSCoV2 podrían también beneficiarse de esta intervención, propiciando orientar la intervención a las necesidades de cada paciente(15).

Con respecto a la intervención en programas de rehabilitación pulmonar, el entrenamiento continuo e interválico en pacientes con reducción significativa de la capacidad funcional, evidencia un incremento en la capacidad funcional y mejor desempeño en sus actividades de la vida diaria(26-28); sin embargo, es necesario que durante el ejercicio sean monitorizadas variables como frecuencia cardiaca, frecuencia respiratoria, $\mathrm{SpO}_{2}$, disnea y percepción de esfuerzo con escala Borg.

Respecto al entrenamiento muscular, el entrenamiento de miembros superiores ofrece reducción de la disnea, sin embargo, no se observan modificaciones de la calidad de vida relacionada con la salud; en otras enfermedades respiratorias crónicas la disfunción de la musculatura inferior contribuye al incremento de la morbimortalidad(29). Sin embargo, pacientes con estancias prolongadas en unidades de cuidado intensivo han mostrado debilidad muscular marcada que impediría la adecuada ejecución del entrenamiento de fuerza y resistencia; es por esto que la electroestimulación neuromuscular (NMES) puede ser una alternativa para intervenir esta población(30).

En general los pacientes con COVID-19 presentan bajos de niveles de oxígeno(31) por lo cual para el entrenamiento de los pacientes debe ser utilizado el soporte de oxígeno, aunque aún no es claro el nivel de saturación mínimo permitido y la mejor forma de administración durante a rehabilitación pulmonar(32).

El impacto psicológico ocasionado por la enfermedad es de alto a severo, y una tercera parte de los pacientes manifiestan ansiedad de moderada a severa, por lo que las intervenciones psicológicas integrales podrían generar un impacto positivo en los pacientes(33) y deben tenerse en cuanta para un tratamiento rehabilitador integral.

Todo programa de rehabilitación pulmonar debería contemplar un componente basado en ejercicio y educativo que permita vincular al paciente y la familia, sin embargo, para minimizar los efectos del distanciamiento social y el asilamiento preventivo, la tele rehabilitación puede ser una estrategia de alto impacto a considerar en aquellos pacientes con mayores dificultades para realizar traslados desde sus hogares(34), en la que se podrían realizar sesiones de ejercicios y educativas individuales/grupales dirigidas en tiempo sincrónico con el fin de mantener los mismos beneficios que la rehabilitación pulmonar convencional realizada en consulta externa ${ }^{34,35) .}$

\section{Conclusiones}

Pacientes con SARSCoV2 en el contexto intrahospitalario pueden beneficiarse de la rehabilitación pulmonar, principalmente con el fin de evitar el deterioro funcional $y$ mejorar la sintomatología. Por otra parte, en el paciente extrahospitalario la rehabilitación pulmonar se indica para mejorar la sintomatología, la capacidad funcional y la calidad de vida. Resulta necesario adoptar recomendaciones y realizar ajustes a los programas de rehabilitación pulmonar que permitan orientar la intervención a un nuevo tipo de patología 
y obtener los mismos beneficios que en otras patologías respiratorias ya conocidas.

\section{Conflicto de intereses: Ninguno declarado por los} autores.

\section{Referencias}

1. Colombia Confirma Su Primer Caso De COVID-19. Colombia: Ministerio de Salud; 2020. Disponible en: https://www.minsalud.gov.co/Paginas/Colombia-confirmasu-primer-caso-de-COVID-19.aspx

2. Juarros L. Protocolo de rehabilitación en planta de pacientes COVID 19. Hospital Universitario 12 de Octubre, Salud Madrid; 2020. Disponible en: https://imas12.es/wpcontent/uploads/2020/Repositorio/02.Protocolo_RHB_pla nta_COVID19_completo.pdf

3. Güell Rous MR, Díaz Lobato S, Rodríguez Trigo G, Morante Vélez F, San Miguel M, Cejudo P, et al. Pulmonary rehabilitation. Arch Bronconeumol. 2014;50(8):332-44. DOI: 10.1016/j.arbres.2014.02.014.

4. Driggin E, Madhavan MV, Bikdeli B, Chuich T, Laracy J, Biondi-Zoccai G, et al. Cardiovascular considerations for patients, health care workers, and health systems during the COVID-19 pandemic. $J$ of American Coll Cardiol. 2020;75(18):2352-71. DOI: 10.1016/j.jacc.2020.03.031.

5. Chan KS, Zheng JP, Mok YW, Li YM, LIU Y-N, Chu CM, et al. SARS: prognosis, outcome and sequelae. Respirology. 2003;8(Suppl 1):S36-40. DOI: 10.1046/j.14401843.2003.00522.x.

6. Ong K-C, Ng AW-K, Lee LS-U, Kaw G, Kwek S-K, Leow MK-S, et al. Pulmonary function and exercise capacity in survivors of severe acute respiratory syndrome. Eur Respir J. 2004;24(3):436-42. DOI: 10.1183/09031936.04.00007104.

7. Moldofsky H, Patcai J. Chronic widespread musculoskeletal pain, fatigue, depression and disordered sleep in chronic post-SARS syndrome; a case-controlled study. BMC Neurol. 2011;11(1):37. DOI: 10.1186/1471-2377-11-37.

8. Xie L, Liu Y, Xiao Y, Tian Q, Fan B, Zhao H, et al. Follow-up study on pulmonary function and lung radiographic changes in rehabilitating severe acute respiratory syndrome patients after discharge. Chest. 2005;127(6):2119-24. DOI: 10.1378/chest.127.6.2119.

9. Ong K-C, Ng AW-K, Lee LS-U, Kaw G, Kwek S-K, Leow MK-S, et al. 1-year pulmonary function and health status in survivors of severe acute respiratory syndrome. Chest. 2005;128(3):1393-400. DOI: 10.1378/chest.128.3.1393.

10. Rooney S, Webster A, Paul L. Systematic Review of Changes and Recovery in Physical Function and Fitness After Severe Acute Respiratory Syndrome-Related Coronavirus Infection: Implications for COVID-19 Rehabilitation. Phys Ther. 2020;100(10):1717-29. DOI: 10.1093/ptj/pzaa129.

11. Frajkova Z, Tedla M, Tedlova E, Suchankova M, Geneid A. Postintubation Dysphagia During COVID-19 OutbreakContemporary Review. Dysphagia. 2020;35(4):549-57. DOI: 10.1007/s00455-020-10139-6.

12. Xie L, Liu Y, Fan B, Xiao Y, Tian Q, Chen L, et al. Dynamic changes of serum SARS-coronavirus IgG, pulmonary function and radiography in patients recovering from SARS after hospital discharge. Respir Res. 2005;6(1):5. DOI: 10.1186/1465-9921-6-5.

13. Desforges M, Le Coupanec A, Dubeau P, Bourgouin A, Lajoie L, Dubé $M$, et al. Human coronaviruses and other respiratory viruses: underestimated opportunistic pathogens of the central nervous system? Viruses. 2020;12(1):14. DOI: 10.3390/v12010014.

14. Mak IWC, Chu CM, Pan PC, Yiu MGC, Ho SC, Chan VL. Risk factors for chronic post-traumatic stress disorder (PTSD) in SARS survivors. Gen Hosp Psychiatry. 2010;32(6):590-8. DOI: 10.1016/j.genhosppsych.2010.07.007.

15. Yang L-L, Yang T. Pulmonary Rehabilitation for Patients with Coronavirus Disease 2019 (COVID-19). Chronic Dis Transl Med. 2020;6(2):79-86. DOI: 10.1016/j.cdtm.2020.05.002.

16. Chinese Association of Rehabilitation Medicine, Respiratory rehabilitation committee of Chinese Association of Rehabilitation Medicine, Cardiopulmonary rehabilitation Group of Chinese Society of Physical Medicine and Rehabilitation. 2019 novel coronavirus pneumonia respiratory rehabilitation guidance (second edition). Chin J Tuberc Respir Dis. 2020;43(4):308-14. DOI: 10.3760/cma.j.cn112147-20200228-00206.

17. Nici L, Donner C, Wouters E, Zuwallack R, Ambrosino N, Bourbeau J, et al. American thoracic society/European respiratory society statement on pulmonary rehabilitation. Am J Respir Crit Care Med. 2006;173(12):1390-413. DOI: 10.1164/rccm.200508-1211ST.

18. Mantha S, Tripuraneni SL, Roizen MF, Fleisher LA. Proposed Modifications in the 6-Minute Walk Test for Potential Application in Patients With Mild COVID-19: A Step to Optimize Triage Guidelines. Anesth Analg. 2020;131(2):398402. DOI: 10.1213/ANE.0000000000004986.

19. Liu K, Zhang W, Yang Y, Zhang J, Li Y, Chen Y. Respiratory rehabilitation in elderly patients with COVID-19: A randomized controlled study. Complement Ther Clin Pract. 2020;39:101166. DOI: 10.1016/j.ctcp.2020.101166.

20. GRUPO PII REHABILITACIÓN RESPIRATORIA, Sociedad española de neumología y cirugía torácica SEPAR. Recomendaciones para el reinicio de rehabilitación respiratoria grupal tras pandemia COVID-19. 2020. Disponible https://drive.google.com/file/d/1faRqlNfvKkomQBXnTzBn 9ozeIYbCkwkh/view

21. Mao L, Jin H, Wang M, Hu Y, Chen S, He Q, et al. Neurologic manifestations of hospitalized patients with coronavirus disease 2019 in Wuhan, China. JAMA Neurol. 2020;77(6):683-90. DOI: 10.1001/jamaneurol.2020.1127.

22. Borg K, Stam H. Covid-19 and Physical and Rehabilitation Medicine. J Rehabil Med. 2020;52(4):JRM00045. DOI: 10.2340/16501977-2679.

23. Tansey CM, Louie M, Loeb M, Gold WL, Muller MP, de Jager J, et al. One-year outcomes and health care utilization in survivors of severe acute respiratory syndrome. Arch Inter Med. 2007;167(12):1312-20. DOI: 10.1001/archinte.167.12.1312.

24. Pulvirenti F, Cinetto F, Milito C, Bonanni L, Pesce AM, Leodori G, et al. Health-Related-Quality of Life in Common Variable Immunodeficiency Italian patients switched to remote assistance during the COVID-19 pandemic. J Allergy Clin Immunol Pract. 2020;8(6):1894-9. DOI: 10.1016/j.jaip.2020.04.003.

25. Klok FA, Boon GJ, Barco S, Endres M, Geelhoed JM, Knauss S, et al. The Post-COVID-19 Functional Status scale: a tool to measure functional status over time after COVID-19. Eur Respir J. 2020;56:2001294. DOI: 10.1183/13993003.014942020.

26. Betancourt-Peña J, Hurtado-Gutiérrez H. Efectos de un programa de rehabilitación pulmonar en pacientes con enfermedad pulmonar Intersticial difusa. Fisioterapia. 2015;37(6):286-92. DOI: 10.1016/j.ft.2014.12.001. 
27. Torres-del Castillo N, Paredes L, Shek N, Hurtado-Gutierrez $\mathrm{H}$, Betancourt-Peña J. Benefits of Pulmonary Rehabilitation in Patients With Idiopathic Pulmonary Fibrosis and Other Interstitial Lung Diseases. Chest. 2017;152(4):A982. DOI: 10.1016/j.chest.2017.08.1018.

28. Wickerson L, Brooks D, Granton J, Reid WD, Rozenberg D, Singer LG, et al. Interval aerobic exercise in individuals with advanced interstitial lung disease: a feasibility study. Physiother Theor Pr. 2019;37(9):1034-42. DOI: 10.1080/09593985.2019.1678207.

29. Iepsen UW, Jørgensen KJ, Ringbæk T, Hansen $H$, Skrubbeltrang C, Lange P. A combination of resistance and endurance training increases leg muscle strength in COPD: an evidence-based recommendation based on systematic review with meta-analyses. Chron Respir Dis. 2015;12(2):132-45. DOI: 10.1177/1479972315575318.

30. Maffiuletti NA, Gondin J, Place N, Stevens-Lapsley J, Vivodtzev I, Minetto MA. Clinical use of neuromuscular electrical stimulation for neuromuscular rehabilitation: what are we overlooking? Arch Phys Med Rehab. 2018;99(4):806-12. DOI: 10.1016/j.apmr.2017.10.028.
31. Mo P, Xing Y, Xiao Y, Deng L, Zhao Q, Wang H, et al. Clinical characteristics of refractory Coronavirus disease 2019 in Wuhan, China. Clinical Infectious Diseases. 2021;73(11):e4208-e4213. DOI: 10.1093/cid/ciaa270.

32. Lyons $\mathrm{C}$, Callaghan $\mathrm{M}$. The use of high-flow nasal oxygen in COVID - 19. Anaesthesia. 2020;75(7):843-47. DOI: 10.1111/anae.15073.

33. Wang C, Pan R, Wan X, Tan Y, Xu L, Ho CS, Ho RC. Immediate psychological responses and associated factors during the initial stage of the 2019 coronavirus disease (COVID-19) epidemic among the general population in China. Intl $J$ Environ Res Public Health. 2020;17(5):1729. DOI: 10.3390/ijerph17051729.

34. Hansen H, Bieler T, Beyer N, Kallemose T, Wilcke JT, Østergaard LM, et al. Supervised pulmonary telerehabilitation versus pulmonary rehabilitation in severe COPD: a randomised multicentre trial. Thorax. 2020;75(5):413-21. DOI: 10.1136/thoraxjnl-2019-214246.

35. Jácome C, Marques A, Oliveira A, Rodrigues LV, Sanches I. Pulmonary telerehabilitation: An international call for action. Pulmonology. 2020;26(6):335-7. DOI: 10.1016/j.pulmoe.2020.05.018. 